\title{
Status of the ANAIS experiment at Canfranc
}

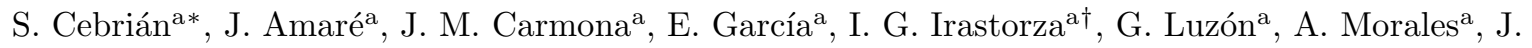 \\ Morales $^{\mathrm{a}}$, A. Ortiz de Solórzano ${ }^{\mathrm{a}}$,J. Puimedón ${ }^{\mathrm{a}}$, M.L. Sarsa ${ }^{\mathrm{a}}$, J. A. Villar ${ }^{\mathrm{a}}$ \\ ${ }^{a}$ Laboratory of Nuclear and High Energy Physics, University of Zaragoza, 50009 Zaragoza, Spain
}

The present status of the ANAIS experiment (Annual Modulation with NaI's) is shown. ANAIS is intended to use more than $100 \mathrm{~kg}$ of $\mathrm{NaI}(\mathrm{Tl})$ in the Canfranc Underground Laboratory (Spain) searching for seasonal modulation effects in the WIMP signal; in a first stage, a prototype (one single $10.7 \mathrm{~kg}$ crystal) has been developed in order to obtain the best conditions regarding the energy threshold and the radioactive background in the low energy region as well as to check the stability of the environmental conditions. The first results corresponding to an exposure of $2069.85 \mathrm{~kg}$ day show an average background level of 1.2 counts/(keV $\mathrm{kg}$ day) from threshold $\left(E_{t h r} \sim 4 \mathrm{keV}\right.$, even using one single photomultiplier) up to $10 \mathrm{keV}$.

\section{INTRODUCTION}

According to current robust evidences from supernovae and the CMB radiation, only $\sim 30 \%$ of the density of the Universe must be due to matter and the rest should consist of unknown species of dark energy. In addition, most of the matter must be dark, non-baryonic and cold. Axions and Weakly Interacting Massive Particles, the socalled WIMPs, are the leading candidates to this type of dark matter. The lightest stable particles of supersymmetric theories, like the neutralino, describe a particular class of WIMPs.

Direct searches of WIMPs, which are supposedly filling the galactic halo, are based on the measurement of the nuclear recoil induced by the elastic scattering off target nuclei in a suitable detector [1]. This process is rare (interaction rates range from 10 to $10^{-5} \mathrm{c} /(\mathrm{kg}$ day $\left.)\right)$ and has an energy spectrum which decays almost exponentially from a few $\mathrm{keV}$; therefore, ultra-low background conditions and very good energy thresholds are mandatory in the direct detection of WIMPs. But even in these conditions, the WIMP signal is entangled with the radioactive background; consequently, to confirm the existence of these particles it would be necessary to identify a distinc-

\footnotetext{
*Attending speaker: scebrian@posta.unizar.es

${ }^{\dagger}$ Present address: CERN, EP Division, CH-1211 Geneva 23, Switzerland
}

tive signature such as the annual modulation of the counting rates due to the variation in the relative velocity between the Earth and the halo, produced by the movement of the Earth around the Sun [2]. The smallness of this effect $(<10 \%)$ makes necessary to accumulate as much statistics as possible having a long time exposure and a large mass of target nuclei, requirement which may be fulfilled using NaI. The DAMA collaboration has reported an annual modulation effect [3] which singles out a region of WIMPs in the parametric space $\sigma-m_{W}$, partially excluded by other experiments (CDMS [曰], EDELWEISS [5], IGEX [6]) and ZEPLIN [7].

\section{THE PROTOTYPE OF ANAIS}

ANAIS (Annual Modulation with NaI's) is a large mass experiment intended to investigate the annual modulation effect in the signal of galactic WIMPs [8]. It will be installed in the Canfranc Underground Laboratory, located in an old railway tunnel in the Spanish Pyrenees with an overburden of 2450 m.w.e., using $\sim 100 \mathrm{~kg}$ of $\mathrm{NaI}(\mathrm{Tl})$ as an improved scaled-up version of a previous experience [9]. Before setting-up the whole experiment, a prototype is being developed in Canfranc in an attempt to obtain the best energy threshold and lowest radioactive background in the low energy region, as well as to check the stability of 
the environmental conditions which influence on the detector response.

In the ANAIS prototype, one out of the 14 $\mathrm{NaI}(\mathrm{Tl})$ detectors stored underground since 1988 has been used; it consists of an hexagonal $10.7 \mathrm{~kg}$ crystal encapsulated inside 0.5 -mm-thick stainless steel and coupled to a photomultiplier (PMT) through a quartz window. Some components of the PMT have been removed because of their radioimpurities. The scintillator has been placed in a shielding consisting of $10 \mathrm{~cm}$ of archaeological lead (of less than $9 \mathrm{mBq} / \mathrm{kg}$ of ${ }^{210} \mathrm{~Pb}$ ) followed by $20 \mathrm{~cm}$ of low activity lead, a sealed box in PVC (maintained at overpressure to prevent the intrusion of radon), 2-mm-thick cadmium sheets, and finally, $40 \mathrm{~cm}$ of polyethylene and tanks of borated water. An active muon-veto made of plastic scintillators is covering the set-up.

The data acquisition system, based on standard NIM and CAMAC electronics, has two different parts following the two output signals implemented from the PMT; the fast signal is recorded using a digital oscilloscope (500 time bins, 10 $\mathrm{ns} /$ bin) while the slow signal is routed through a linear amplifier and analog-to-digital converters controlled by a PC through parallel interfaces, to register the energy of events up to $\sim 1.7 \mathrm{MeV}$.

\subsection{Noise rejection}

The noise in NaI detectors is mainly produced by thermoionic emission in photomultipliers. The different shape of the output signals from the PMT for scintillation and noise events makes feasible an efficient discrimination. Figure 1 shows a typical noise pulse and the theoretical shapes of scintillation and noise for a pulse having the same area. Typical parameters of the PMT pulses used in other $\mathrm{NaI}$ experiments to reject the noise are the mean amplitude [10], a ratio of area portions 11], etc. In the present work, the filtering of noise uses the squared deviation $d$ of the digitalized pulse $V_{p}$ from the well-known theoretical shape $V$ of a scintillation event of the same area:

$$
\begin{aligned}
& d=100 \times \frac{\sum_{i}\left(V\left(t_{i}\right)-V_{p}\left(t_{i}\right)\right)^{2}}{\sum_{i} V_{p}\left(t_{i}\right)} \\
& V(t)=-\frac{Q R}{\tau-\tau_{s}}\left(\exp \left(-t / \tau_{s}\right)-\exp (-t / \tau)\right)
\end{aligned}
$$

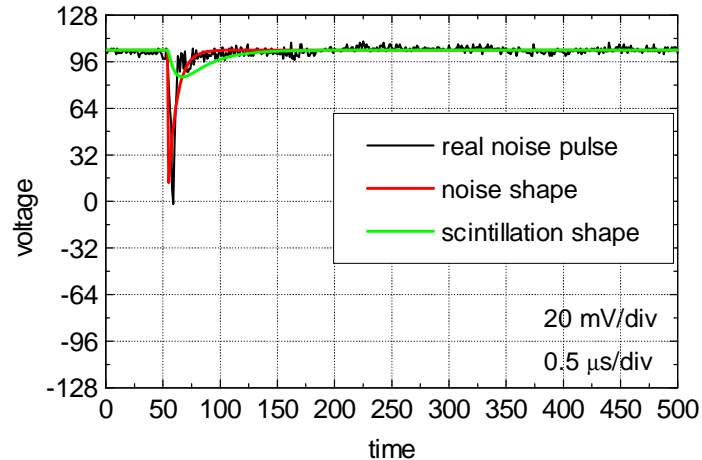

Figure 1. Output pulse from the PMT for a typical noise event and the theoretical shapes of noise and scintillation for a pulse having the same area.

where $\tau=R C$ is the time constant of the $\mathrm{RC}$ circuit equivalent to the PMT, $\tau_{s}$ is the scintillation decay constant $(\sim 230 \mathrm{~ns}$ for $\mathrm{NaI}(\mathrm{Tl}))$ and $Q$ is the total collected charge. To eliminate the noise, a safe cut at $3 \sigma$ from the center of the gaussian distribution of this parameter for a population of ${ }^{137} \mathrm{Cs}$ calibration events from 4 to $10 \mathrm{keV}$ has been used. The effect of the noise rejection can be seen in Figure 2, where the raw spectrum and the spectrum after the elimination of noise are depicted up to $100 \mathrm{keV}$.

\subsection{Radioactive background}

By comparing the data recorded from December 2000 to October 2001 with Monte Carlo simulations, it was possible to identify the main sources of background in the region of interest. The ${ }^{210} \mathrm{~Pb} 46.5 \mathrm{keV}$ line as well as a peak due to the escape of X-rays of I at $\sim 16 \mathrm{keV}$ seen in the spectrum (see Figure 2), may be caused by the presence of radioimpurities in the stainless steel can and/or in the PMT. The area of the $1460.8 \mathrm{keV}$ peak is compatible with an activity of $15 \mathrm{mBq} / \mathrm{kg}$ from ${ }^{40} \mathrm{~K}$ in the NaI crystal; these contaminations produce an almost flat 


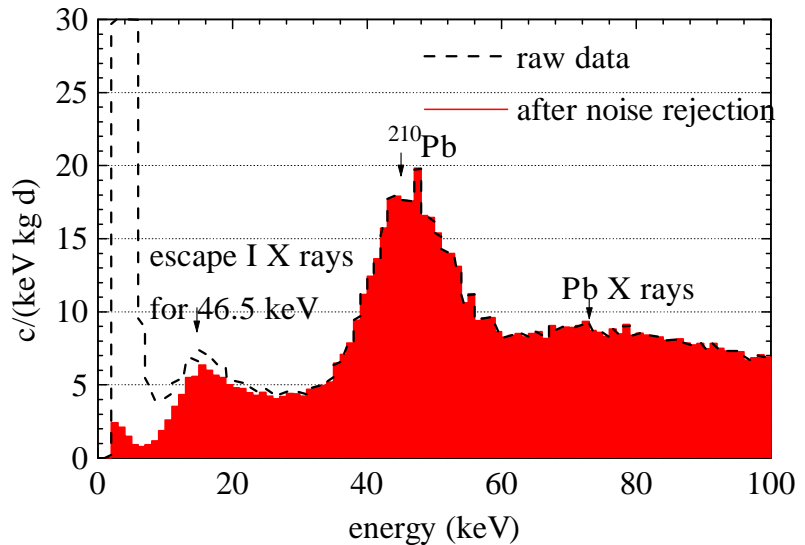

Figure 2. Low-energy region of the observed spectrum in the prototype of ANAIS before and after the noise rejection.

background in the low energy region due to their beta emission. It is also worth noting that a comparison between the spectra recorded with and without the neutron shielding does not show noticeable differences.

A pulse shape analysis has been carried out in the low energy region with the purpose of investigating the possible appearance of the socalled "anomalous" or "bump" events found in other $\mathrm{NaI}$ experiments 12,13. No evidence of such anomaly has been discovered in the distributions for background events, neither following the method of the UKDMC (fitting integrated pulses to calculate the decay time constant) nor using other parameters (like the first momemtum of the pulse).

\subsection{Stability}

In an experiment searching for a very small seasonal modulation, the stability of the conditions whose variation could mimic the effect we are looking for is essential. A monitoring system will be installed in ANAIS to control periodically,

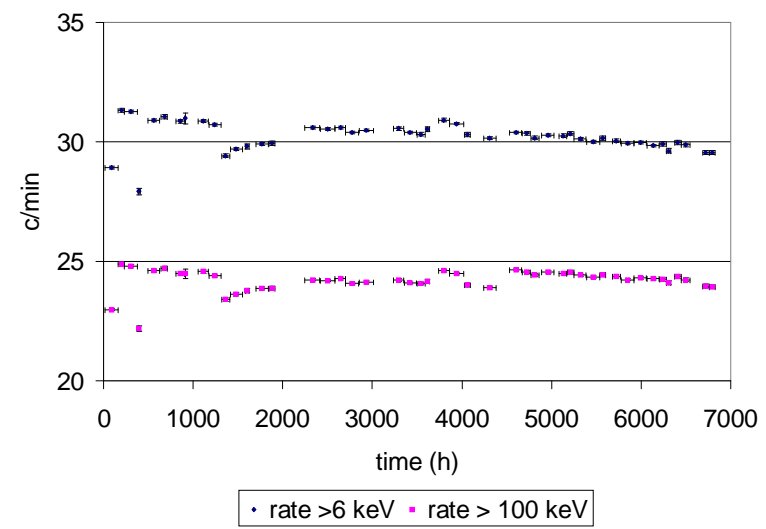

Figure 3. Evolution in time for the whole data collection of the counting rates integrated from 6 $\mathrm{keV}$ and from $100 \mathrm{keV}$.

and even modify automatically, the environmental conditions in the laboratory. But using the data of the prototype, collected along almost 7000 hours, the stability of some parameters has been already checked.

The fluctuations of the ADC channels for peaks used to calibrate energy spectra are $\sim 1-1.5 \%$, so the variations in the energy of events due to this effect are negligible compared to the energy resolution. The evolution in time of different counting rates (total, above $6 \mathrm{keV}$ and above $100 \mathrm{keV}$ ) has been checked, since a modulation effect in the low energy region due to background (not to WIMPs) should be correlated with a modulation at higher energies. These rates are plotted as a function of time for the whole data collection in Figure 3. The gaussian distributions of the deviations of the rates from their mean values have a sigma of 1.75 for the rate above $6 \mathrm{keV}$, and 1.58 for the rate above $100 \mathrm{keV}$.

Since the scintillation light yield depends on temperature, a monitoring system (based on sensors and ADAM sensor-to-computer interfaces) has been tentatively developed to study the tem- 
perature in the inner enceinte. Internal temperature does not depend neither on changes on the nitrogen gas flux injected inside the shielding nor on the periodical fluctuations of the temperature in the laboratory due to the air conditioning system. It is only sensitive to real changes outside the shielding. If a good stability of the external temperature is achieved, variations inside will be of only 0.03 Celsius degrees.

\section{RESULTS}

The results presented here correspond to an exposure of $2069.85 \mathrm{~kg} \times$ day. As pointed out before, Figure 2 shows the raw spectrum and the spectrum after the noise rejection up to $100 \mathrm{keV}$. The energy threshold is of $\sim 4 \mathrm{keV}$ and the background level registered from the threshold up to $10 \mathrm{keV}$ is about 1.2 counts/( $\mathrm{keV} \mathrm{kg}$ day).

We have used this region to derive the corresponding limits for the WIMP-nucleon cross sections. The galactic halo is supposed to be isotropic, isothermal and non-rotating, assuming a density of $\rho=0.3 \mathrm{GeV} / \mathrm{cm}^{3}$, a Maxwellian velocity distribution with $v_{r m s}=270 \mathrm{~km} / \mathrm{s}$ (with an upper cut corresponding to an escape velocity of $650 \mathrm{~km} / \mathrm{s}$ ) and a relative Earth-halo velocity of $v_{r}=230 \mathrm{~km} / \mathrm{s}$. The Helm parameterization [14] is used for the coherent form factor, while the approximation from 15] is considered for the SD case. Spin factors $\left(\lambda_{p} J(J+1)\right)$ 0.089 and 0.126 are assumed for $\mathrm{Na}$ and I respectively. Fig. 1 shows, in addition to the limits derived from the prototype results (solid lines), the estimates considering a flat background of 1 count/(keV $\mathrm{kg}$ day) from 2 to $8 \mathrm{keV}$ after an exposure of $107 \mathrm{~kg} \times \mathrm{y}$ both for raw data (dotted lines) and assuming Pulse Shape Discrimination (PSD) techniques (dashed lines); rejection factors obtained on the average by other groups have been used [16]. The plots show the contour lines for each nucleus, $\mathrm{Na}$ and I, as well as the $\mathrm{NaI}$ case. That is shown both for SI scalar interactions (top) and SD WIMP-proton interactions (bottom). It should be noted that for SI interactions and using PSD techniques, ANAIS will be able to explore the region of WIMPs singled out by the possible annual modulation effect reported by the DAMA collaboration [3], even though it is not designed to be an exclusion experiment.
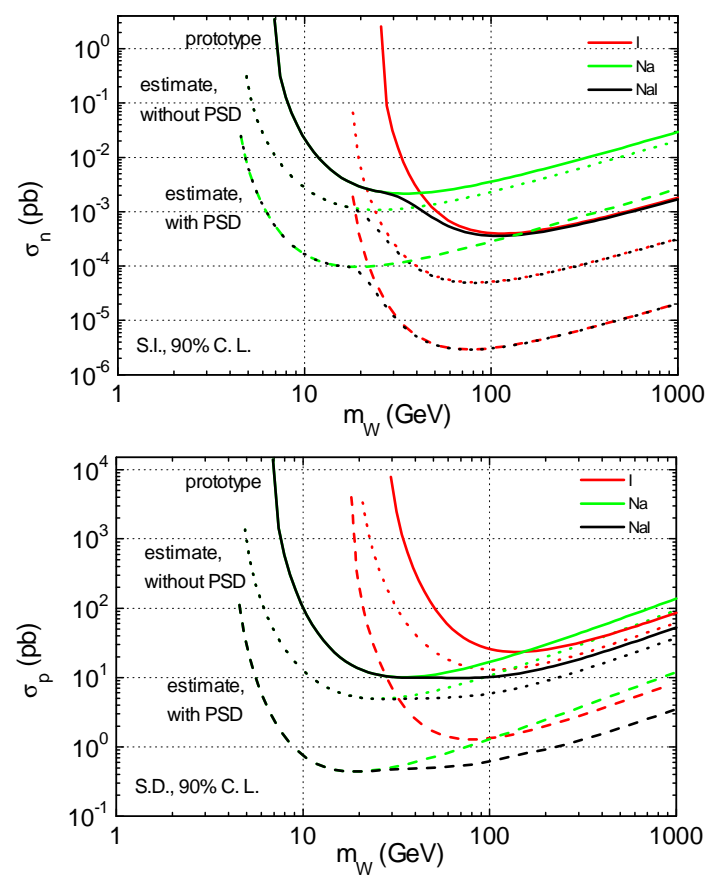

Figure 4. Exclusion plots derived for SI (top) and SD (bottom) interactions from the prototype (solid line) and expected for the whole experiment with (dashed line) and without (dotted line) PSD techniques.

According to these first results, the next steps in the development of the prototype of ANAIS, currently underway, are the removal of the present PMT and the steel can so as to install, instead, two ultra-low background PMT and a 1cm-thick teflon enclosure filled with special mineral oil, as in the NAIAD experiment [17. A program of measurements to select high radiopurity materials has been carried out in Canfranc, using an ultra-low background Ge detector. The goals of these modifications are to reduce, as much as 
possible, the various sources of background, to diminish the noise by using anticoincidence readout (lowering so also the energy threshold) and to improve the collection of the scintillation light.

\section{CONCLUSIONS}

Summarizing, a prototype of the ANAIS experiment has been installed in the Canfranc Underground Laboratory. The noise rejection has lead to a $\sim 4 \mathrm{keV}$ threshold, even using a single PMT. The background in the low energy region is produced by ${ }^{210} \mathrm{~Pb}$ in the detector enclosure and/or the PMT and ${ }^{40} K$ in the $\mathrm{NaI}(\mathrm{Tl})$ crystal. The stability is being controlled and new improvements are being developed before setting-up the large-mass experiment.

\section{ACKNOWLEDGEMENTS}

The Canfranc Astroparticle Underground Laboratory is operated by the University of Zaragoza under contract No. FPA2001-2437. This research was funded by the Spanish Commission for Science and Technology (CICYT) and the Government of Aragón.

\section{REFERENCES}

1. For recent surveys of WIMP detection, see for instance: A. Morales, Nucl. Phys. B (Proc. Suppl.) 87 (2000) 477 astro-ph/9912554 and Review Talk at the TAUP 2001 Workshop, LNGS, (September 2001), Nucl. Phys. B (Proc. Suppl.) 110 (2002) 39 astro$\mathrm{ph} / 0112550$.

2. A. K. Drukier, K. Freese and D. N. Spergel. Phys. Rev. D 33 (1986) 3495. K. Freese, J. Frieman and A. Gould. Phys. Rev. D 37 (1988) 3388.

3. R. Bernabei et al. Phys. Lett. B 480 (2000)23.

4. R. Abusaidi et al. [CDMS Collaboration], Phys. Rev. Lett. 84 (2000) 5699 astroph/0002471]. D. Abrams et al. [CDMS Collaboration], submitted to Phys. Rev. D astro$\mathrm{ph} / 0203500$.

5. A. Benoit et al. [EDELWEISS Collaboration], Phys. Lett. B 513 (2001) 15 astroph/0106094]. A. Benoit et al. [EDELWEISS
Collaboration], submitted to Phys. Lett. B astro-ph/0206271.

6. A. Morales et al. [IGEX Collaboration], Phys. Lett. B 532 (2002) 8 hep-ex/0110061].

7. V.A.Kudryavtsev et al. [Boulby Dark Matter Collaboration], Poster Session at Neutrino 2002, Munich (May 2002). To appear in Nucl. Phys. B (Proc. Suppl.).

8. S. Cebrián et al. Nucl. Phys. B (Proc. Suppl.) 110 (2002) 94 [hep-ex/0111075].

9. M. L. Sarsa et al. Phys. Rev. D 56 (1997) 1856.

10. G. Gerbier et al. Astrop. Phys. 11 (1999) 287.

11. R. Bernabei et al. Il Nuovo Cimento A 112 (1999) 545.

12. I. Liubarsky et al. Nucl. Phys. B (Proc. Suppl.) 87 (2000) 64.

13. G. Gerbier et al. Nucl. Phys. B (Proc. Suppl.) 87 (2000) 61.

14. J. Engel. Phys. Lett. B 264 (1991) 114.

15. J. D. Lewin y P. F. Smith. Astrop. Phys. 6 (1996) 87.

16. R. Bernabei et al. Phys. Lett. B 389 (1996) 757. P. F. Smith et al. Phys. Lett. B 379 (1996) 299.

17. N. J. C. Spooner et al. Phys. Lett. B 473 (2000) 330. 